\title{
Study of Optimal Sizing for Residential Sorption Heat Pump System
}

\author{
Corey BLACKMAN ${ }^{1}$, Kyle R. GLUESENKAMP ${ }^{2 *}$, Mini MALHOTRA ${ }^{2}$, Zhiyao YANG $^{2}$ \\ ${ }^{1}$ SaltX Technology, Hägersten, Stockholm, Sweden \\ ${ }^{2}$ Oak Ridge National Laboratory, Oak Ridge, Tennessee, USA
}

*Corresponding Author
gluesenkampk@ornl.gov

\begin{abstract}
Gas-driven sorption heat pumps (GDSHP) show significant potential to reduce primary energy use, associated emissions and energy costs for space heating and domestic hot water (DHW) production in residential applications. In this study a bivalent system was considered, characterised by the integration of a novel modular sorption heat pump component and a condensing boiler. The modular heat pump component, or sorption module (SM), has been developed in two types: Type A and Type B, either of which could be integrated into a bivalent GDSHP system. The Type A sorption module had a functioning principle based on a solid chemisorption cycle, while Type B operates under a resorption cycle. To investigate the applicability of each SM type, a bivalent GDSHP system with a Type A SM (GDSHPA) and one with a Type B SM (GDSHPB) were evaluated. Simulations of year-round space heating loads for two single family houses, one in New York and the other Minnesota, were carried out and the seasonal gas coefficient of performance (SGCOP) for each GDSHP system calculated. The impact of the ratio of the design heating capacity of the SM compared to the peak heating capacity of the bivalent GDSHP was studied. Results show that SGCOP was not significantly affected for SM design heating capacity ratios greater than 66\% of the peak GDSHPA design capacity in Minnesota, and 21\% for GDSHPB. In New York, the ratios were 55\% and 35\% for GDSHPA and GDSHPB respectively.
\end{abstract}

\section{INTRODUCTION}

Sorption heat pumps may use the combustion of natural gas as a heat source to drive a sorption cycle. This cycle effectively pumps heat from a low temperature source to one of higher temperature allowing for more effective gas utilisation for space and domestic water heating. Cycle efficiencies as high as $140 \%$ (i.e. COP 1.4) for gasdriven sorption heat pumps (GDSHP) are not uncommon [1]. A bivalent or hybrid GDSHP integrates a sorption component with a condensing boiler. Bivalent GDSHPs are typically dimensioned such that the supplementary boiler meets peak heating demand of the building. It should be noted that, in general, these peak demand conditions occur only for relatively few hours of the year. Therefore, the more costly sorption module should be dimensioned to avoid inefficient operation at low part loads, while cost effectively maximising the SGCOP. This opens the possibility of cost-effective GDSHPs tailor-sized for specific climates. Highly modular sorption heat pump components known as sorption modules (SM) have been developed for integration into GDSHPs. Two module types with different sorption cycle principles were experimentally evaluated. One SM based on solid chemisorption cycle principle (type A) and the other a resorption cycle (type B), both employing ammonia as the working fluid. The details of the experimental evaluation are beyond the scope of this study. For this study, simplified performance correlations for each SM type were used to determine the optimum capacity of the SM when implemented in a bivalent GDSHP. Two climate zones in the USA were studied, representative of a cold (Minnesota) and a moderate (New York) climate.

\section{METHODOLOGY}

Hourly building space heating load data was generated via simulations with a 2006 IECC code compliant EnergyPlus residential prototype model for New York and Minnesota. A bin method was utilised in which outdoor temperatures, heating demand of the building and required heating supply temperatures were used to calculate annual heating energies and efficiencies. The data were evaluated in two concurrent evaluation sets with inputs and outputs shown in Figure 1. Evaluations were done for each SM type for 4 scenarios: $1-$ low fuel prices and cold climate, 2 - low fuel prices and moderate climate, 3 - high fuel prices and cold climate, 4 high fuel prices and moderate climate. 


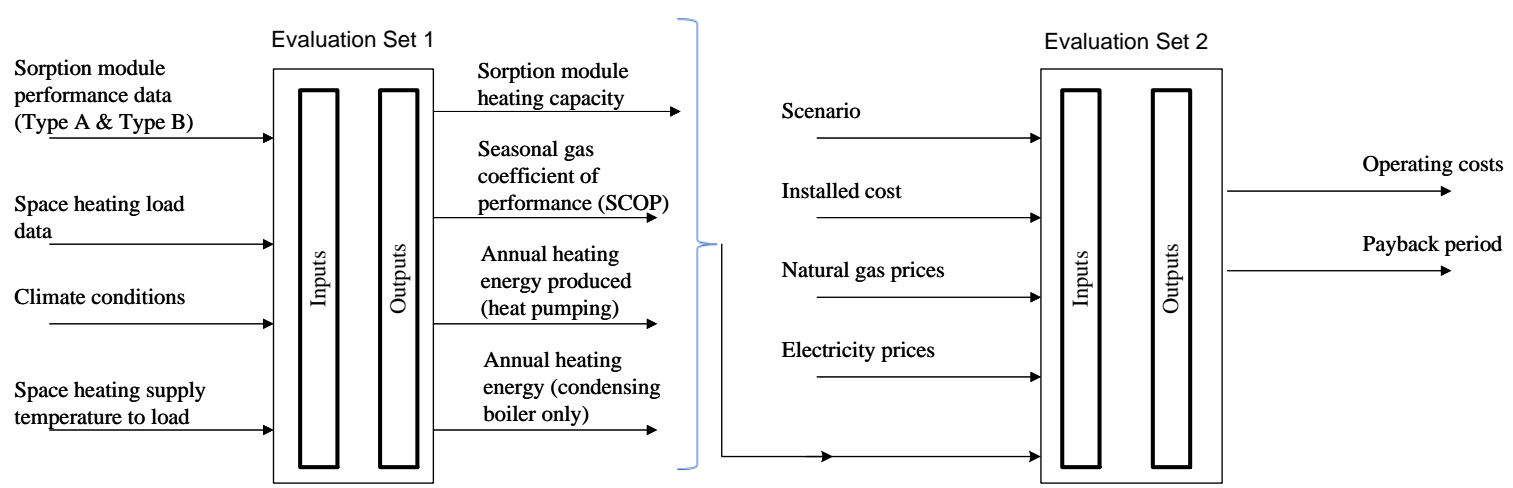

Figure 1 - Schematic diagram showing data input and output flow for evaluation of optimum sorption module capacity.

\section{RESULTS AND DISCUSSION}

Results from the evaluations are shown in Figure 2.

New York - SGCOP vs Heating Capacity Ratio

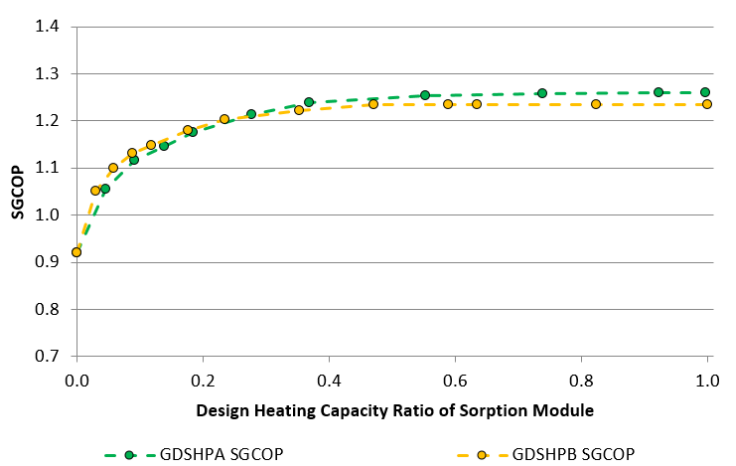

New York - Payback Period vs Heating Capacity Ratio

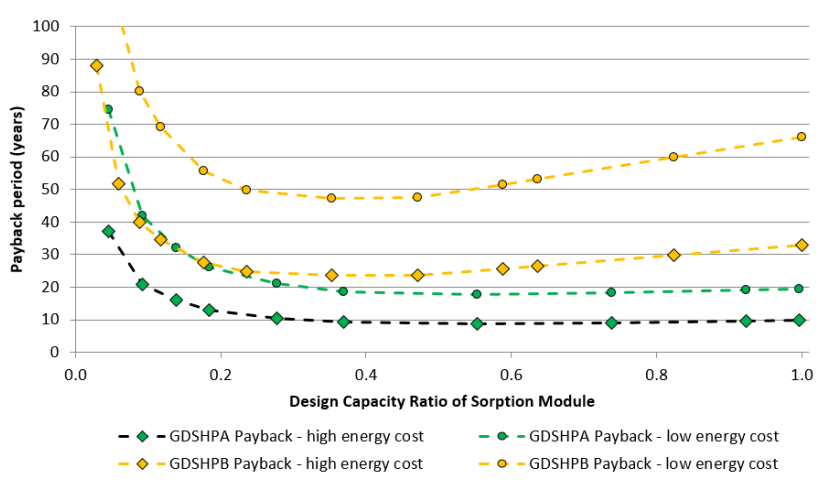

Figure 2 - SGCOP (left) and simple payback period (right) versus SM design heating capacity ratio for New York.

Longer payback periods are observed for the GDSHPB compared to GDSHPA under all conditions in both climates. This is attributed to the higher expected cost of the Type B SM. Despite having higher cycle COPs at ambient temperatures above $10^{\circ} \mathrm{C}$, the GDSHPB had a lower SGCOP due to a limited range of operation.

\section{CONCLUSIONS AND PERSPECTIVES}

The results of the evaluation indicated that the sorption module design heating capacity ratio that results in the shortest payback period is 0.55 and 0.35 for GDSHPA and GDSHPB respectively, for systems installed in New York. In the case of Minnesota, the corresponding optimum capacity ratios were 0.66 for GDSHPA and 0.28 for GDSHPB. These sorption module sizes resulted in at least $97 \%$ of maximum achievable SGCOP. In New York, a GDSHPA, with SM capacity ratio of 55\%, had expected payback periods of 17.8 years and 8.9 years for the high energy cost and low energy cost scenarios respectively. The GDSHPB system, even at optimum SM capacity ratios, exhibited payback periods longer than 20 years for both energy cost scenarios.

\section{ACKNOWLEDGMENTS}

This work was sponsored by the U. S. Department of Energy's Building Technologies (BTO) Office under Contract No. DE-AC05-00OR22725 with UT-Battelle, LLC. The authors also acknowledge Mr. Antonio Bouza, Technology Manager for HVAC\&R, Water Heating, and Appliance, U.S. Department of Energy BTO.

\section{REFERENCES}

[1] Aprile M, Scoccia R, Toppi T, Guerra M, Motta M. Modelling and experimental analysis of a GAX NH3-H2O gas-driven absorption heat pump. Int J Refrig. 2016;66:145-55.

This manuscript has been authored by UT-Battelle, LLC under Contract No. DE-AC05-00OR22725 with the U.S. Department of Energy. The United States Government retains and the publisher, by accepting the article for publication, acknowledges that the United States Government retains a non-exclusive, paidup, irrevocable, world-wide license to publish or reproduce the published form of this manuscript, or allow others to do so, for United States Government purposes. The Department of Energy will provide public access to these results of federally sponsored research in accordance with the DOE Public Access Plan (http://energy.gov/downloads/doe-public-access-plan).

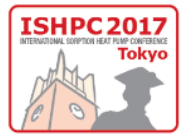

